\title{
Forming Collaborations through the Data Quality Committee to Address Urgent Incidents
}

\author{
Sophia Crossen ${ }^{2}$, Krystal S. Collier ${ }^{1}$, Courtney Fitzgerald ${ }^{3}$, Kaitlyn Ciampaglio ${ }^{4}$, Lakshmi \\ Radhakrishnan ${ }^{5}$, Jill Baber 6 \\ ${ }^{1}$ Public Health Statistics, Arizona Department of Health Services, Phoenix, Arizona, United States, ${ }^{2}$ Kansas Department of Environment and Health, \\ Topeka, Kansas, United States, ${ }^{3}$ Cerner Corporation, Kansas City, Missouri, United States, ${ }^{4}$ Division of Health Informatics and Surveillance, Centers \\ for Disease Control and Prevention, Atlanta, Georgia, United States, ${ }^{5}$ McKing Consulting Corporation, Atlanta, Georgia, United States, ${ }^{6}$ North Dakota \\ Department of Health, Bismark, North Dakota, United States
}

\section{Objective}

The National Syndromic Surveillance Program (NSSP) Community of Practice (CoP) works to support syndromic surveillance by providing guidance and assistance to help resolve data issues and foster relationships between jurisdictions, stakeholders, and vendors. During this presentation, we will highlight the value of collaboration through the International Society for Disease Surveillance (ISDS) Data Quality Committee (DQC) between jurisdictional sites conducting syndromic surveillance, the Centers for Disease Control and Prevention's (CDC) NSSP, and electronic health record (EHR) vendors when vendor-specific errors are identified, using a recent incident to illustrate and discuss how this collaboration can work to address suspected data anomalies.

\section{Introduction}

On November 20, 2017, several sites participating in the NSSP reported anomalies in their syndromic data. Upon review, it was found that between November 17-18, an EHR vendor's syndromic product experienced an outage and errors in processing data. The ISDS DQC, NSSP, a large EHR vendor, and many of the affected sites worked together to identify the core issues, evaluate ramifications, and formulate solutions to provide to the entire NSSP CoP.

\section{Description}

Syndromic surveillance site administrators in several jurisdictions noticed data anomalies after running regular daily and weekend trend reports on November 20, 2017, including large amounts of either missing or extra data. After investigating the data further, they were able to narrow their focus to locations using one common EHR vendor. Some sites also noticed similar trends in electronic laboratory reporting data. While the anomalies were not consistent across or even within sites, these issues were reported to the NSSP, which supported early awareness and response efforts. The EHR vendor resources became aware early on November 18 th when internal data quality reports contained irregularly large amounts of data. Beginning on Monday November 20th the EHR vendor team followed procedures for notifying clients and public health programs of the issue via emails and posting to shared community forums. The routine DQC meeting on December 8, 2017 included a brief discussion of the incident, bringing this to the attention of community members not yet aware of the issue or not sure how to proceed in correcting errors in their data.

The NSSP engaged its own CDC-based site inspectors to review data trends for all facilities and work with sites to identify facilities using the EHR vendor product so they could better target their investigations. The inspectors also sent a notice to all site administrators informing the community of potential anomalies in their data. This prompted site administrators nationwide to evaluate data for facilities using the vendor product, and review communications sent by vendor representatives to some of the affected sites. The NSSP then provided information to sites regarding the process for deleting data from the NSSP servers and ESSENCE.

To address ongoing questions and confusion that persisted throughout the community, the DQC held another meeting on January 12, 2018 to bring sites, the NSSP, and EHR vendor representatives together to discuss how to correct data anomalies. Solutions were proposed and potential drawbacks for each were discussed. Because the impact to each site was somewhat unique, multiple solutions were employed based on site specific concerns, capabilities, and requirements. 
Acknowledgement

Centers for Disease Control, Cerner Corporation, Data Quality Committee 